\title{
Magnetic Fluids' Heating Power Exposed to a High-Frequency Rotating Magnetic Field
}

\author{
Miloš Beković (D), Mislav Trbušić, Mladen Trlep, Marko Jesenik, and Anton Hamler \\ Faculty of Electrical Engineering and Computer Science, University of Maribor, Maribor, Slovenia \\ Correspondence should be addressed to Miloš Beković; milos.bekovic@um.si
}

Received 18 August 2017; Revised 8 December 2017; Accepted 16 December 2017; Published 31 January 2018

Academic Editor: Gianluca Gubbiotti

Copyright (C) 2018 Miloš Beković et al. This is an open access article distributed under the Creative Commons Attribution License, which permits unrestricted use, distribution, and reproduction in any medium, provided the original work is properly cited.

\begin{abstract}
Magnetic fluids are superparamagnetic materials that have recently been the subject of extensive research because of their unique properties. Among them is the heating effect when exposed to an alternating magnetic field, wherein the objective is to use this property in medicine as an alternative method for the treatment of tumors in the body. The heating effect characterization for the alternating magnetic field (AMF) has been studied widely, whilst for the rotational magnetic field (RMF), no systematic study has been done yet. In this article, we present the characterization of the heating power of magnetic fluids in a high-frequency rotational magnetic field. The results show similar behavior of heating power or specific absorption rate characteristics as in AMF.
\end{abstract}

\section{Introduction}

Magnetic fluids are stable colloidal suspensions of superparamagnetic nanoparticles in a carrier liquid and have been the subject of numerous researches due to their specific properties. Their specific characteristics attracted attention when they were subjected to a time-changing magnetic field, which may have pulsating form (alternating magnetic field $(\mathrm{AMF})$ ) or rotary form (rotational magnetic field (RMF)).

This article will highlight the behavior of the magnetic fluid in the RMF, which has been at the forefront of research since 1980, in which Popplewell et al. [1] were interested in the rotational motion of the liquid due to low-frequency magnetic fields.

More recently, applications of magnetic fluids and magnetic nanoparticles have penetrated increasingly into the field of biomedicine, as proposed in [2]. Possible fields of application include magnetic resonance imaging, cell sorting, drug delivery, magnetic hyperthermia, and others [3].

The latter represents a medical treatment where a magnetic-fluid tumor-loaded tissue is exposed to a highfrequency alternating magnetic field with the aim of raising the temperature of the diseased tissues. An ideal hyperthermia treatment should destroy the tumor cells selectively without damaging the surrounding healthy tissue. This can be achieved with an increase in the temperature of the tissue to a value between 41 and $43^{\circ} \mathrm{C}$. The limitations of such a process, advantages, and disadvantages are gathered in $[4,5]$.

Magnetic fluids exposed to AMF show immense heating effect, which is a direct consequence of the different physical mechanisms of conversion of the energy of a magnetic field into heat. The intensity of the heating depends strongly on the parameters of the magnetic field (frequency, amplitude, field homogeneity, etc.), as well as the structure of the magnetic fluid (particle size distribution and its type, carrier liquid, surfactant, etc.). Changing these parameters and measurement methods is fairly well known, and their summary is found in [6-10].

The behavior of magnetic fluid exposed to the RMF is not new, since already in 1999 Lacis [11] attempted to demonstrate the movement of fluid in certain field frequencies. For frequencies up to $40 \mathrm{~Hz}$, an s-shape, or spiral, formation of the ferrofluid has also been investigated $[12,13]$.

Dieckhoff et al. [14] had been studying the behavior of magnetic nanoparticles exposed to RMF and AMF at low frequencies by means of phase-lag research. This would also be possible with the system used in this research, but the amplitude of the magnetic field is too small for accurate determination of magnetic field intensity. 


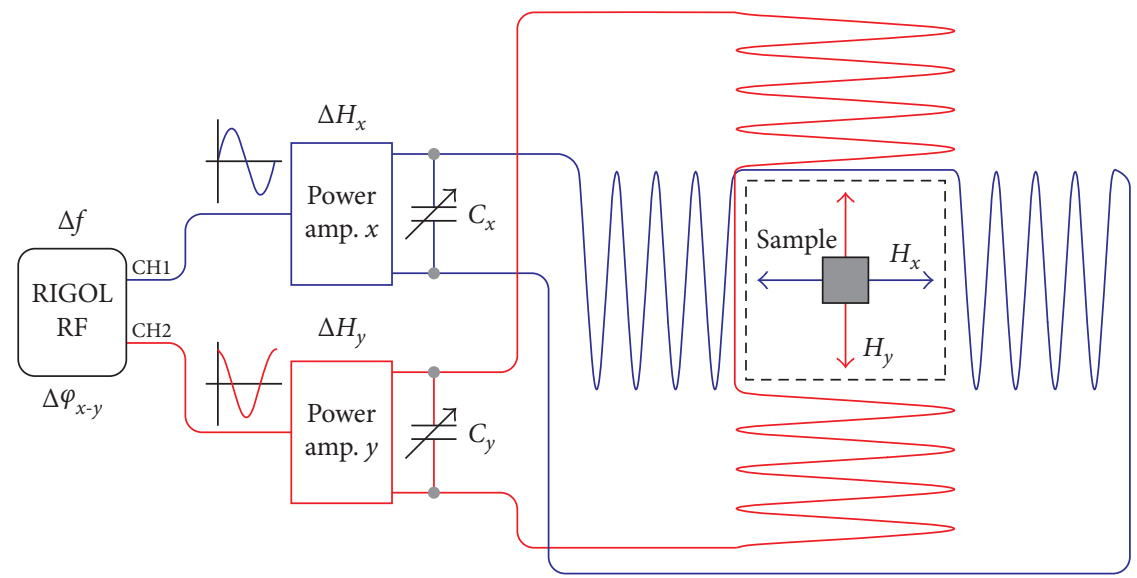

FIGURE 1: Arrangement of the supply coils of the measurement system and the position of the sample in the center.

It is also possible to simulate the dynamic behavior of particles in a magnetic field solving Fokker-Planck equations, as was done in $[14,15]$, and the results have been verified experimentally, again only for low frequencies. The basis for the construction of an RMF was taken from [13] and [15-17] and modified in such a way that magnetic fields could be generated suitable for medical hyperthermia.

In this paper, a new measuring system is presented for generating an RMF of high frequencies and satisfactory amplitudes, suitable for medical hyperthermia. The main novelty of this article is the systematic characterization of magnetic flux losses in both types of magnetic fields (AMF and RMF), where we have confirmed experimentally the thesis given in [18]. The results of the analysis and improvement of the measurement system for a better determination of the magnetic field are presented in the following sections.

\section{Theory of Power Dissipation}

Cantillon-Murphy et al. [19] extended the theory of magnetic fluid losses from Rosensweig [18], who studied the physical mechanisms of heat generation in a time-changing magnetic field. Heat is caused by the delay in relaxation of the magnetic moment through either the rotation within the particle or the rotation of the particle itself. The first mechanism is called the Néel relaxation and the second one the Brownian relaxation. Relaxation times of a particle are given in (1) for the Néel relaxation $\left(\tau_{\mathrm{N}}\right)$ and in (2) for the Brownian relaxation $\left(\tau_{\mathrm{B}}\right)$ :

$$
\begin{gathered}
\tau_{\mathrm{N}}=\tau_{0} e^{K_{\mathrm{a}} V_{\mathrm{p}} / k T}, \\
\tau_{\mathrm{B}}=\frac{3 V_{\mathrm{h}} \eta}{k T}
\end{gathered}
$$

where $\tau_{0}$ is the characteristic time constant $10^{-9}, K_{\mathrm{a}}$ is the anisotropy constant, $V_{\mathrm{p}}$ is the volume of the magnetic nanoparticle, $k$ is Boltzmann's constant, $T$ is the temperature, $V_{\mathrm{h}}$ is the hydrodynamic volume of the magnetic

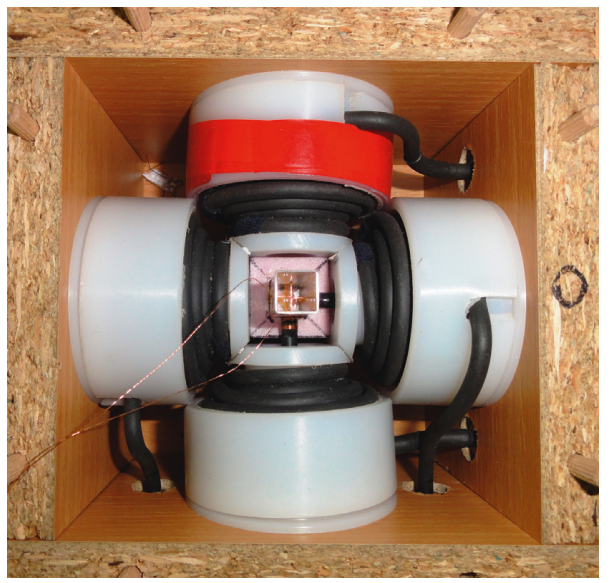

Figure 2: Actual measurement system in a $20 \times 20 \mathrm{~cm}$ box.

nanoparticle and surfactant, and $\eta$ is the dynamic viscosity of the carrier fluid.

Magnetic relaxation of magnetic nanoparticles is derived from the Shilomis equation, and relaxation time $\tau$ constant depends on the particle size, where the Brownian relaxation dominates the large particles, whilst the Néel relaxation dominates smaller particles:

$$
\tau=\frac{\tau_{\mathrm{B}} \tau_{\mathrm{N}}}{\tau_{\mathrm{B}}+\tau_{\mathrm{N}}}
$$

The maximum value of chord susceptibility $\chi$ is fielddependent magnetic susceptibility, and it is calculated in the following equation:

$$
\chi=\frac{\phi M_{d}}{\widehat{H}}\left(\operatorname{coth} \alpha-\frac{1}{\alpha}\right) ; \quad \alpha=\frac{\mu_{0} V_{\mathrm{p}} M_{d}}{k T},
$$

where it is strongly dependent on the amplitude of the exposed magnetic field, $\widehat{H}$; volume fraction of magnetic nanoparticles in fluid, $\phi$; the saturation magnetization of bulk material, $M_{d}$ (in the case of magnetite $445 \mathrm{kA} / \mathrm{m}$ ); and the permeability of free space, $\mu_{0}$.

When magnetic fluid is exposed to a rotational magnetic field, a vector of magnetic field strength can be divided into $x$ 


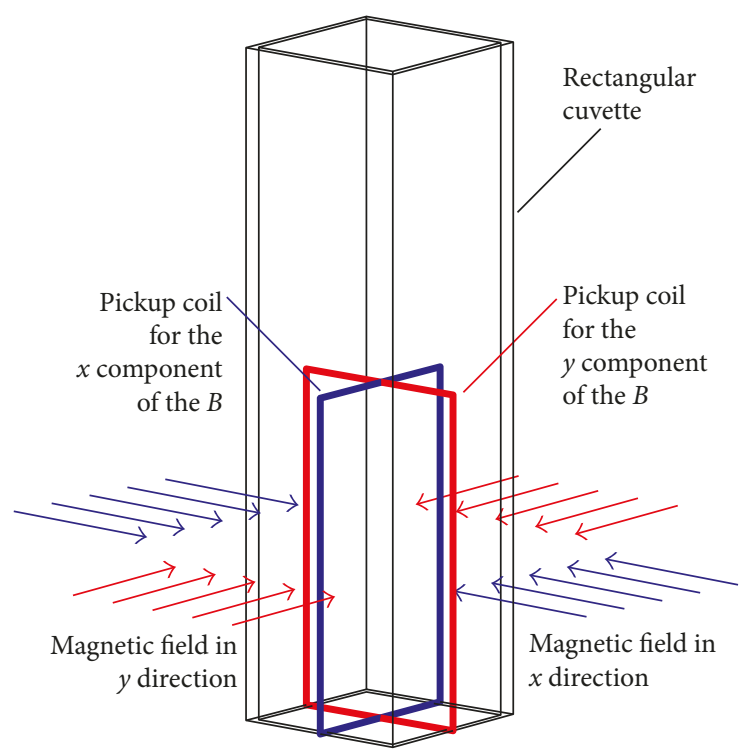

(a)

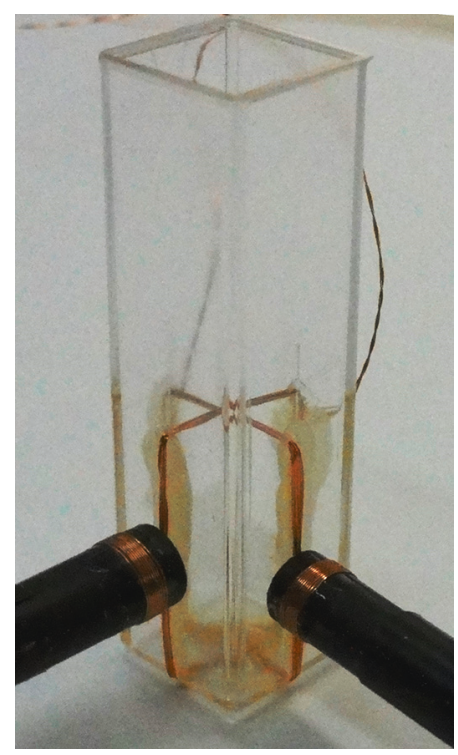

(b)

FIGURE 3: (a) Scheme for the sample cuvette adapted to contain a pair of orthogonal coils with 5 turns each for the $x$ and $y$ directions. (b) The actual picture of all four-measurement coils outside the system.

and $y$ components, $H_{x}$ and $H_{y}$, and also the magnetization components $\hat{m}$ can be separated into the $x$ and $y$ directions and calculated by the following equation:

$$
\begin{aligned}
& \widehat{m}_{x}=\chi \frac{\widehat{H}_{x}}{(\Omega \tau+1)}, \\
& \widehat{m}_{y}=\chi \frac{\widehat{H}_{y}}{(\Omega \tau+1)},
\end{aligned}
$$

where $\Omega$ is the circular frequency of magnetic field $(2 \pi f)$. In the case of $s$ purely alternating magnetic field, one of the field components is set to zero, either $H_{x}$ or $H_{y}$, whilst in a rotational magnetic field both amplitudes are the same.

The heating power of the specific absorption rate (SAR), or the power dissipation $P$, is derived from the physical properties of the fluid and the variables of the rotating magnetic field from [19], and it corresponds to the following equation:

$$
P=\mu_{0}\left(\frac{\Omega}{2}\left(\widehat{m}_{x} H_{x}+\widehat{m}_{y} H_{y}\right)\right) \text {. }
$$

\section{Rotating Magnetic Field}

3.1. Supply Coil Assembly. The easiest RMF can be generated with a rotating magnet, but such systems rarely exceed the frequency of $50 \mathrm{~Hz}$, which is completely useless for the application; in addition, the magnetic field strength cannot be changed. Another approach to creating RMF is with a stator of a three-phase induction motor, where locally distributed coils are powered with the appropriate time-shifted voltages, as is done in [17]. This approach is not applicable for this application because of two problems. The first one is frequency-exponential growth of magnetic core losses in regard to $50 \mathrm{~Hz}$ that the core is built for. In our frequency range $(\mathrm{kHz})$, this would produce a lot of heat; hence, it is unusable. The second problem is the heating of supply coils, as, due to the eddy current effect, they are also highly dependent on the frequency. The solution of the first problem is to use a set of coils without a magnetic core, where the coils should have significantly larger turns, or increase the current to achieve satisfactory magnetic conditions. The other problem is solved by using a coil made from copper pipes, connected to the cooling water which dissipates the heat produced by eddy currents and, at the same time, allows substantially higher currents and, consequently, greater magnetic field amplitudes. The basic distribution of supply coils is seen in Figure 1, where two half coils are perpendicular to each other. This represents a two Helmholtz coil pair.

The system has an RF function generator, RIGOL DG4102, which allows a dual supply for two power generators. The power signals can be changed in amplitude and phase delay arbitrarily. This solution enables a stable power supply and the appropriate $90^{\circ}$ phase shift to create an RMF.

For this system, we use two AC power amplifiers operating independently of each other. Their output powers are $2.0 \mathrm{~kW}$ and $0.7 \mathrm{~kW}$ and, hence, the weaker one limits their maximal current.

The objective of system planning was to maximize the amplitude of $H$ by varying the geometry of the coils. An analysis of the coils' parameter (height, width, diameter, and number of layers) impact on the $H$ is made in [20], whilst the actual picture of the three-layered system of half coils is presented in Figure 2. In the center of the system is a rectangular cuvette for the sample, while two round measurement coils are placed on the right and bottom.

3.2. Measurement System. The precise determining of the magnetic field plays an important role in the characterization 


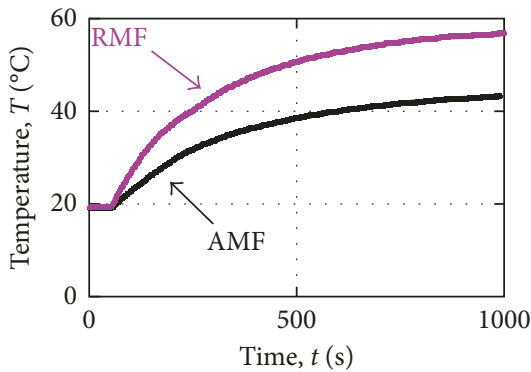

$H=2.0 \mathrm{kA} / \mathrm{m}$

$f=244 \mathrm{kHz}$

(a)

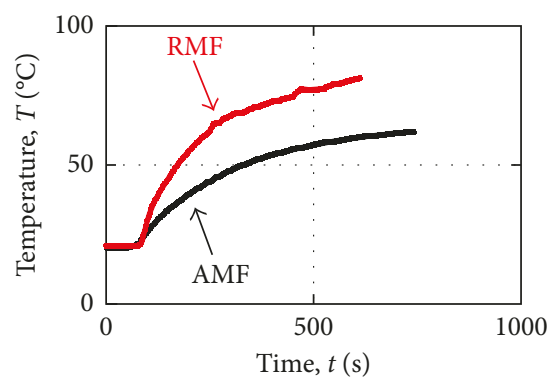

$H=2.0 \mathrm{kA} / \mathrm{m}$

$f=504 \mathrm{kHz}$

(c)

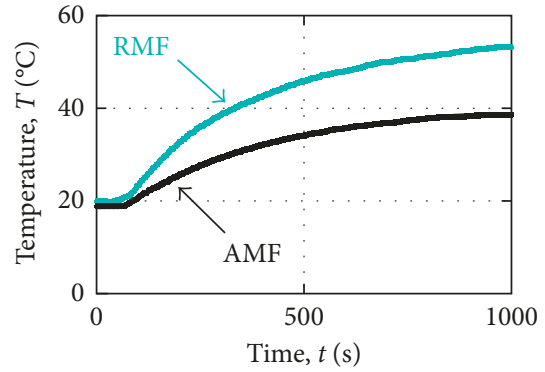

$H=1.0 \mathrm{kA} / \mathrm{m}$

$f=671 \mathrm{kHz}$

(b)

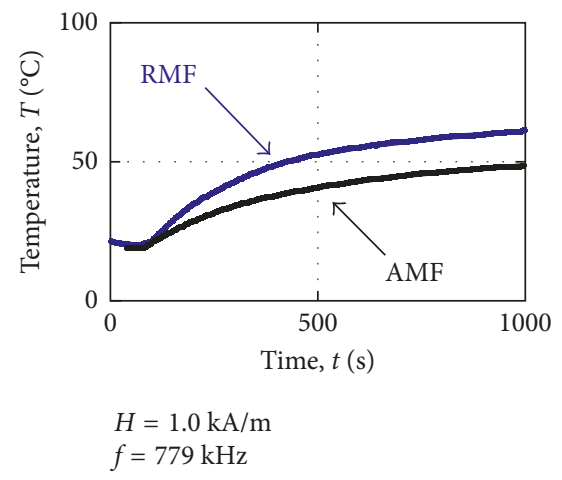

(d)

FIgURE 4: Comparison of the heating of the magnetic fluid sample exposed to AMF and RMF at different frequencies and amplitudes.

of magnetic fluids. An important modification according to [20] represents the determination of the parameters of the magnetic field $b(t)$ and $h(t)$. Field assessment from the supply current and the numerical calculations of the field were replaced with actual measurements, where we added two pairs of measurement coils in both $x$ and $y$ directions.

The first pair is two round measurement coils that can be seen in Figure 3(b), and they measure the induced voltages in air $e_{h-r}$ close to the sample. For determination of $h(t)$ from (7), the geometric properties of the coil are needed, cross section $A_{h-r}$ and number of turns $N_{h-r}$, where index " $r$ " refers to the round shape.

The second pair is two quadratic-shaped coils that measure the induced voltages $e_{b-q}$ for determination of the flux density $b(t)$ from (8). Due to their shape, they are denoted with index " $q$," whilst their geometric properties of cross section $A_{b-q}$ and number of turns $N_{b-q}$ must be known:

$$
\begin{aligned}
& h(t)=\frac{1}{\mu_{0} N_{h-r} A_{h-r}} \int e_{h-r}(t) d t, \\
& b(t)=\frac{1}{N_{b-q} A_{b-q}} \int e_{b-q}(t) d t .
\end{aligned}
$$

An alternative method for determining the loss of magnetic fluids in the magnetic field is a method of magnetic measurement [8-10]. For example, AMF works well compared to the conventional method of SAR. Zeng [21] determined magnetic losses of magnetorheological fluids in 2D RMF of low frequencies. They achieved relatively highamplitude fields and plotted $\mathrm{BH}$ vector loci. Therefore, for plotting $\mathrm{BH}$ loci of magnetic fluids in the RMF, we need described measuring coils so that we can determine the components of magnetic flux density in both directions $b_{x}(t)$ and $b_{y}(t)$ and magnetic field strengths $h_{x}(t)$ and $h_{y}(t)$. Losses $P$ in the RMF are calculated using the following equation:

$$
P=\frac{1}{\rho T} \cdot\left(\int_{0}^{T} h_{x}(t) \frac{d b_{x}(t)}{d t} d t+\int_{0}^{T} h_{y}(t) \frac{d b_{y}(t)}{d t} d t\right),
$$

where $\rho$ is the density.

From the research with the AMF, we know that the more pronounced hysteresis loop appears in fields above $5 \mathrm{kA} / \mathrm{m}$ for frequencies above $100 \mathrm{kHz}$; therefore, we only received inadequate results with respect to output constraints on the RMF system. Hence, this theory will be confirmed as soon as we provide larger fields.

\section{Results and Discussion}

4.1. Magnetic Fluid Sample. Since this is a continuation of the research, we use here the sample magnetic fluid as in [20]. The fluid was composed of mineral oil as the carrier liquid and maghemite $\gamma-\mathrm{Fe}_{2} \mathrm{O}_{3}$ nanoparticles with a mean diameter of $10.9 \mathrm{~nm}$. Saturation magnetization was $M_{\mathrm{s}}=42.3 \mathrm{kA} / \mathrm{m}$, 


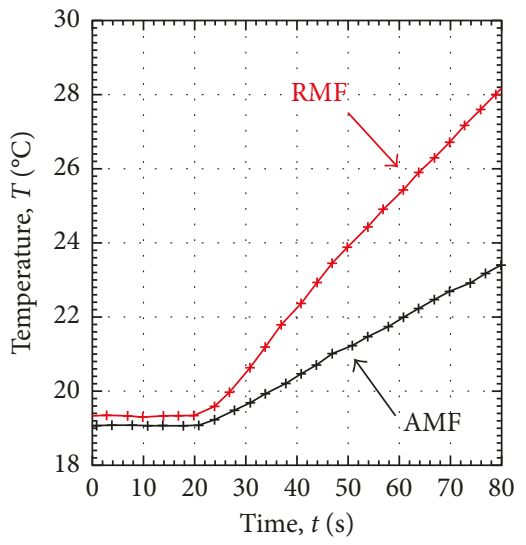

(a)

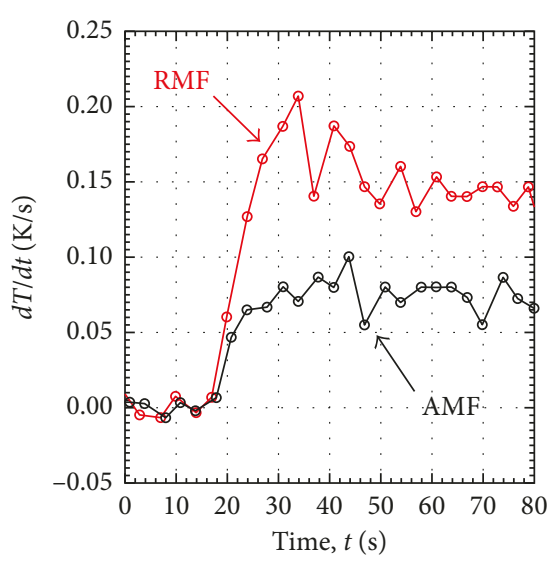

(b)

FIGURE 5: (a) Temperature of first $80 \mathrm{~s}$ of heating for $244 \mathrm{kHz}$ and $2 \mathrm{kA} / \mathrm{m}$ AMF and RMF, and (b) its corresponding time derivatives.

TABLE 1: Maximal temperature derivative: comparison of AMF and RMF.

\begin{tabular}{lcccc}
\hline$f(\mathrm{kHz})$ & $H(\mathrm{kA} / \mathrm{m})$ & $\mathrm{AMF}(d T / d t)_{\max }(\mathrm{K} / \mathrm{s})$ & $\mathrm{RMF}(d T / d t)_{\max }(\mathrm{K} / \mathrm{s})$ & $\mathrm{RMF} / \mathrm{AMF}$ \\
\hline 130 & 4.1 & 0.1333 & 0.2733 & 0.5267 \\
160 & 4.1 & 0.2401 & 0.2067 & 2.1937 \\
244 & 2.0 & 0.1000 & 0.6667 & 2.0670 \\
504 & 2.0 & 0.2467 & 0.1267 & 2.7025 \\
671 & 1.0 & 0.0733 & 0.1667 & 1.7285 \\
779 & 1.0 & 0.1133 & & 1.4713 \\
\hline
\end{tabular}

which revealed an iron concentration of $10.57 \%$. For the test, we used an undiluted sample of $2.0 \mathrm{ml}$ volume.

SAR values are determined using the well-known equation (10) where the measured initial slope of the heating curve is the only variable besides the constant parameters of the fluid, specific heat, $C_{\mathrm{f}}$, mass of the fluid, $m_{\mathrm{f}}$, and the mass of the iron particles, $m_{\mathrm{Fe}}$ :

$$
\mathrm{SAR}=\frac{C_{\mathrm{f}} m_{\mathrm{f}}}{m_{\mathrm{Fe}}}\left(\frac{d T}{d t}\right)_{\text {max }} .
$$

4.2. Rotating and Alternating Magnetic Field. In [20], we have checked the difference between the two types of fields and, for two frequencies, made the thesis that the loss of the RMF is approximately twice as high as in the AMF. Due to the more stable power supply in the case of an RMF and easier control of phase shift, the thesis has been tested further for higher frequencies and came to similar conclusions as those proposed in theory (4), where heating power $P$ and specific SAR are equivalent. The time course of the fluid heating for a single or dual supply is presented in Figure 4 for four examples; in all, it is evidently more pronounced warming for the RMF, which was to be expected.

However, since time courses are incomparable with each other, a maximum temperature derivative has been found for all examples, which is also the key to the loss or SAR evaluation. For this, only a first minute of heating curve is relevant where heating is at most intense as seen in Figure 5(a).
Figure 5(b) shows temperature derivatives of both curves, where maximum values needed for SAR evaluation are clearly shown.

The results for all measurements are summarized in Table 1, where, in the last column (AMF/RMF), the maximum $d T / d t$ ratio was calculated. In most cases, double the value of losses in the case of RMF can be seen, except for the last two measuring points, where slightly smaller values are calculated, which is possible for several reasons. The reasons for the deviation of the results are smaller deviations of the manually adjusted system excitation parameters in case of RMF, among which are the phase angle changes between signals and the amplitude inequalities of $x$ and $y$ signals. Nevertheless, we conclude that the losses double in a wider frequency band.

4.3. SAR Characterization in RMF. Measurement of losses in the RMF at a constant amplitude and frequency poses a major challenge, as it is necessary to regulate the three variables. The frequency in both systems $(x, y)$ is equal and does not change during the measurements, as well as the adjusted phase angle of both trigger signals of the power amplifiers. Due to the use of an LC-resonance circuit, this does not mean the same phase shift in the power section of the circuit but requires a constant adaptation at the signal side. Minor deviations $\left( \pm 5^{\circ}\right)$ from the desired $90^{\circ}$ time lag in power supply signals mean less distorted fields compared to a circular field, whilst increasing deviations results in a more and more elliptical field. 


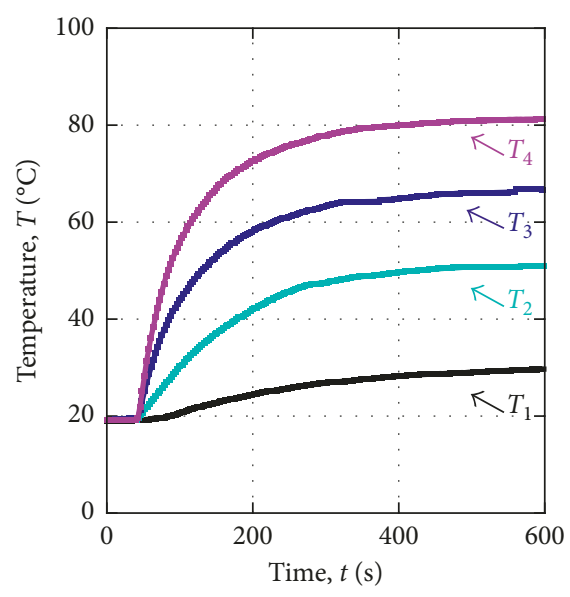

(a)

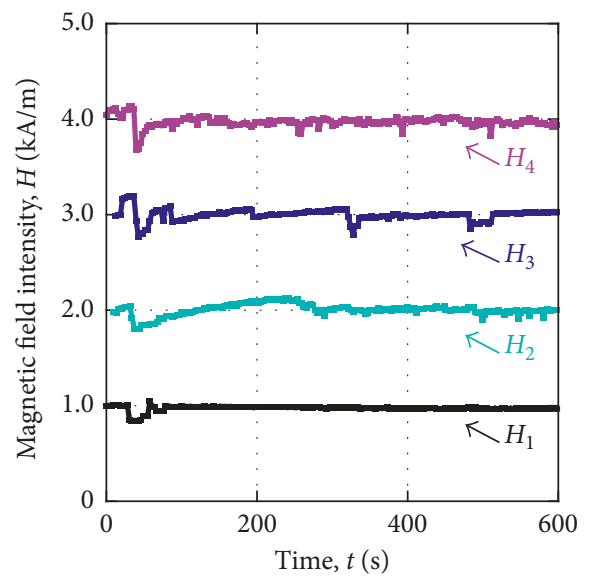

(b)

Figure 6: (a) The time course of temperature for the four RMF examples at a frequency of $100 \mathrm{kHz}$. (b) The time course of the amplitude of the RMF.

Due to the heating of the capacitors, the capacity $C$ varies, and hence, the LC circuit is no longer in resonance. Although the frequency is not changed during the measurement, it was necessary to adjust the amplitude of the currents (the magnetic fields $H_{x}$ and $H_{y}$ ) in such a way that the characterization can be really carried out at a constant $f$ and $H$.

Measurements were carried out in such a way that we first set up capacitors for resonance at the selected frequency and then set the amplitudes $H_{x}$ and $H_{y}$ whilst cooling the supply coils with cooling water. The sample containing the optical temperature sensor was then inserted in the center of the system; the measured temperature time response from the beginning to the steady state is shown in Figure 6(a). Figure 6(b) shows the time courses of the RMF amplitude $H$ and represents the mean value of both the amplitudes $H_{x}$ and $H_{y}$.

The SAR losses at the frequency of $100 \mathrm{kHz}$ in Figure 7 are obtained in such a way that the maximum temperature derivative $(d T / d t)_{\max }$ is determined and multiplied appropriately with the constants according to (10); the result is the desired SAR $=f(H)$ dependence. Similarly, the characterization was performed for frequencies from 200 to $600 \mathrm{kHz}$. It is also evident that, by increasing the frequencies, maximum power output decreased for both the power supplies in such a way that, at $100 \mathrm{kHz}$, a maximum field of $4.5 \mathrm{kA} / \mathrm{m}$ was achieved, whilst at $600 \mathrm{kHz}$, only $1.2 \mathrm{kA} / \mathrm{m}$.

At each frequency, four points were measured, which was enough to determine the approximate function well with adding the point of $(0.0)$.

\section{Conclusion}

In this article, we confirmed experimentally several theses regarding loss of magnetic fluid in a magnetic field. In the previous system, we demonstrated the double loss of only two frequencies $(130$ and $160 \mathrm{kHz})$, since the phase-shiftsignal circuit did not permit higher frequencies; in this work, we confirmed that for frequency spectrum from 100 to

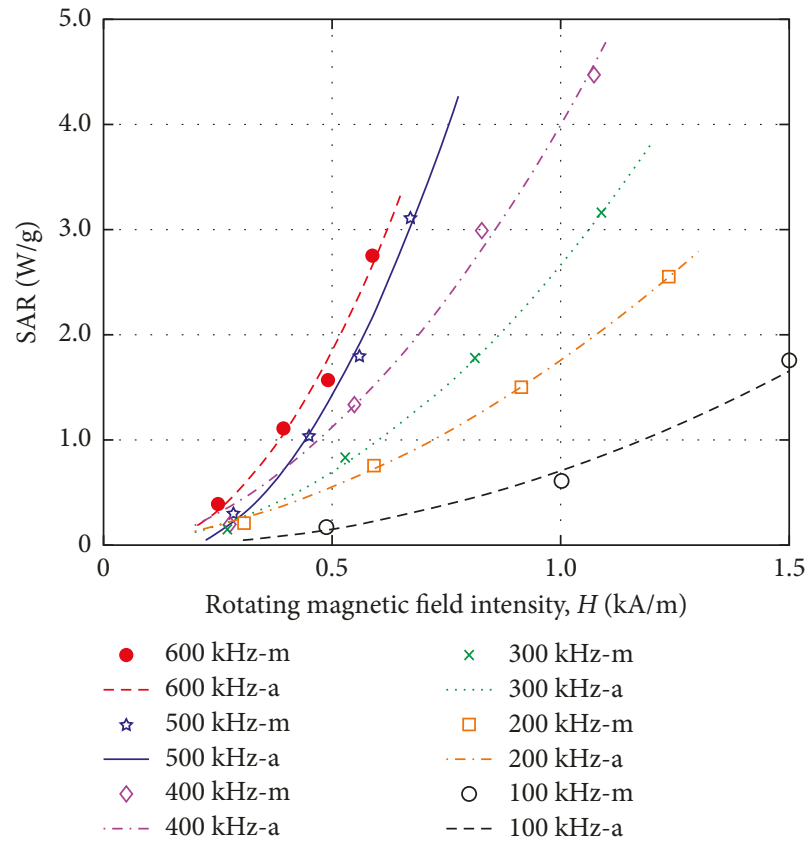

FIGURE 7: SAR of magnetic fluid in RMF for different frequencies, denotation " $m$ " stands for the discrete measured values, whilst "a" is the approximate function for the selected frequency.

$800 \mathrm{kHz}$ and proved the theoretical basis of doubled losses in RMF in comparison with AMF.

Because the sample is situated outside the coil and, therefore, in a relatively low field, we were nevertheless able to show the expected dependence of the $\operatorname{SAR}=f(f, H)$, in the RMF, which is similar to the dependence of the AMF.

Measurement of the characteristics of the magnetic field variables $B$ and $H$ was carried out with a couple of pairmeasurement coils, which allowed a more accurate determination of $B$ and $H$ in the $x$ and $y$ directions.

Due to the low susceptibility of the sample and the relatively small magnetic field amplitudes, we did not see the 
desired nonlinearity in the loci $x y$ curve as in the case of the AMF [8], where hysteresis appeared in the case of field amplitude $5 \mathrm{kA} / \mathrm{m}$ and above.

\section{Conflicts of Interest}

The authors declare that there are no conflicts of interest.

\section{References}

[1] J. Popplewell, R. E. Rosensweig, and R. J. Johnston, "Magnetic field induced rotations in ferrofluid," IEEE Transactions on Magneticsss, vol. 26, no. 5, pp. 1852-1854, 1990.

[2] H. Khurshid, S. Balakrishnan, L. Colak, M. J. Bonder, and G. C. Hadjipanayis, "Water dispersible Fe/Fe-oxide core-shell structured nanoparticles for potential biomedical applications," IEEE Transactions on Magnetics, vol. 45, no. 10, pp. 4877-4879, 2009.

[3] E. Pollert, P. Kašpar, K. Závěta, V. Herynek, M. Burian, and P. Jendelová, "Magnetic nanoparticles for therapy and diagnostics," IEEE Transactions on Magnetics, vol. 49, no. 1, pp. 7-10, 2013.

[4] R. Hegrt, W. Andreä, C.G. d'Ambly et al., "Physical limits of hyperthermia using magnetite fine particles," IEEE Transaction on Magnetics, vol. 34, no. 5, pp. 3745-3754, 1998.

[5] S. Laurenta, S. Dutz, U. O. Häfeli, and M. Mahmoudi, "Magnetic fluid hyperthermia: Focus on superparamagnetic iron oxide nanoparticles," Advances in Colloid and Interface Science, vol. 166, no. 1-2, pp. 8-23, 2011.

[6] D. H. Kim, Y. T. Thai, D. E. Nikles, and C. S. Brazel, "Heating of aqueous dispersions containing $\mathrm{MnFe} 2 \mathrm{O} 4$ nanoparticles by radio-frequency magnetic field induction," IEEE Transactions on Magnetics, vol. 45, no. 1, pp. 64-70, 2009.

[7] A. Candeo and F. Dughiero, "Numerical FEM models for the planning of magnetic induction hyperthermia treatments with nanoparticles," IEEE Transactions on Magnetics, vol. 45, no. 3, pp. 1658-1661, 2009.

[8] M. Beković and A. Hamler, "Determination of the heating effect of magnetic fluid in alternating magnetic field," IEEE Transactions on Magnetics, vol. 46, no. 2, pp. 552-555, 2010.

[9] J. Carrey, B. Mehdaoui, and M. Respaud, "Simple models for dynamic hysteresis loops calculations of magnetic singledomain nanoparticles: application to magnetic hyperthermia optimization," Journal of Applied Physics, vol. 109, no. 8, p. 083921, 2011.

[10] M. Beković, M. Trlep, M. Jesenik, V. Goričan, and A. Hamler, "An experimental study of magnetic-field and temperature dependence on magnetic fluid's heating power," Journal of Magnetism and Magnetic Materials, vol. 331, pp. 264-268, 2013.

[11] S. Lacis, "Bending of ferrofluid droplet in rotating magnetic field," Journal of Magnetism and Magnetic Materials, vol. 201, no. 1-3, pp. 335-338, 1999.

[12] S. Rhodes, J. Perez, S. Elborai, S.-H. Lee, and M. Zahn, "Ferrofluid spiral formations and continuous-to-discrete phase transitions under simultaneously applied DC axial and AC in-plane rotating magnetic fields," Journal of Magnetism and Magnetic Materials, vol. 289, pp. 353-355, 2005.

[13] A. Zakinyan, O. Nechaeva, and Y. Dikansky, "Motion of a deformable drop of magnetic fluid on a solid surface in a rotating magnetic field," Experimental Thermal and Fluid Science, vol. 39, pp. 265-268, 2012.

[14] J. H. Dieckhoff, T. Yoshida, K. Enpuku, M. Schilling, and F. Ludwig, "Homogeneous bioassays based on the manipulation of magnetic nanoparticles by rotating and alternating magnetic fields-a comparison," IEEE Transactions on Magnetics, vol. 48, no. 11, pp. 3792-3795, 2012.

[15] T. Yoshida, K. Enpuku, J. H. Dieckhoff, M. Schilling, and F. Ludwig, "Magnetic fluid dynamics in a rotating magnetic field," Journal of Applied Physics, vol. 111, p. 053901, 2012.

[16] S. Khushrushahi and M. Zahn, "Ultrasound velocimetry of ferrofluid spin-up flow measurements using a spherical coil assembly to impose a uniform rotating magnetic field," Journal of Magnetism and Magnetic Materials, vol. 323, no. 10, pp. 1302-1308, 2011.

[17] P. Hajiani and F. Larachi, "Controlling lateral nanomixing and velocity profile of dilute ferrofluid capillary flows in uniform stationary, oscillating and rotating magnetic fields," Chemical Engineering Journal, vol. 223, pp. 454-466, 2013.

[18] R. E. Rosensweig, "Heating magnetic fluid with alternating magnetic field," Journal of Magnetism and Magnetic Materials, vol. 252, pp. 370-374, 2002.

[19] P. Cantillon-Murphy, L.L. Wald, E. Adalsteinsson, and M. Zahn, "Heating in the MRI environment due to superparamagnetic fluid suspensions in a rotating magnetic field," Journal of Magnetism and Magnetic Materials, vol. 322, no. 6, pp. 727-733, 2010.

[20] M. Bekovi, M. Trlep, M. Jesenik, and A. Hamler, "A comparison of heating effect of magnetic fluid between the alternating and rotating magnetic field," Journal of Magnetism and Magnetic Materials, vol. 355, pp. 12-17, 2014.

[21] J. B. Zeng, "Magnetic hysteresis property of magnetorheological fluid material under 2D fluxes," in Proceedings of 2011 IEEE International Conference on Applied Superconductivity and Electromagnetic Devices, Sidney, Australia, December 2011. 


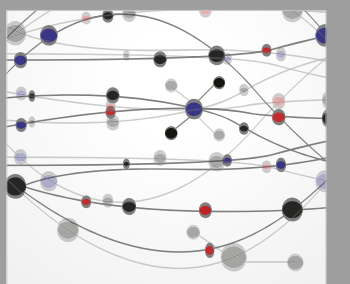

The Scientific World Journal
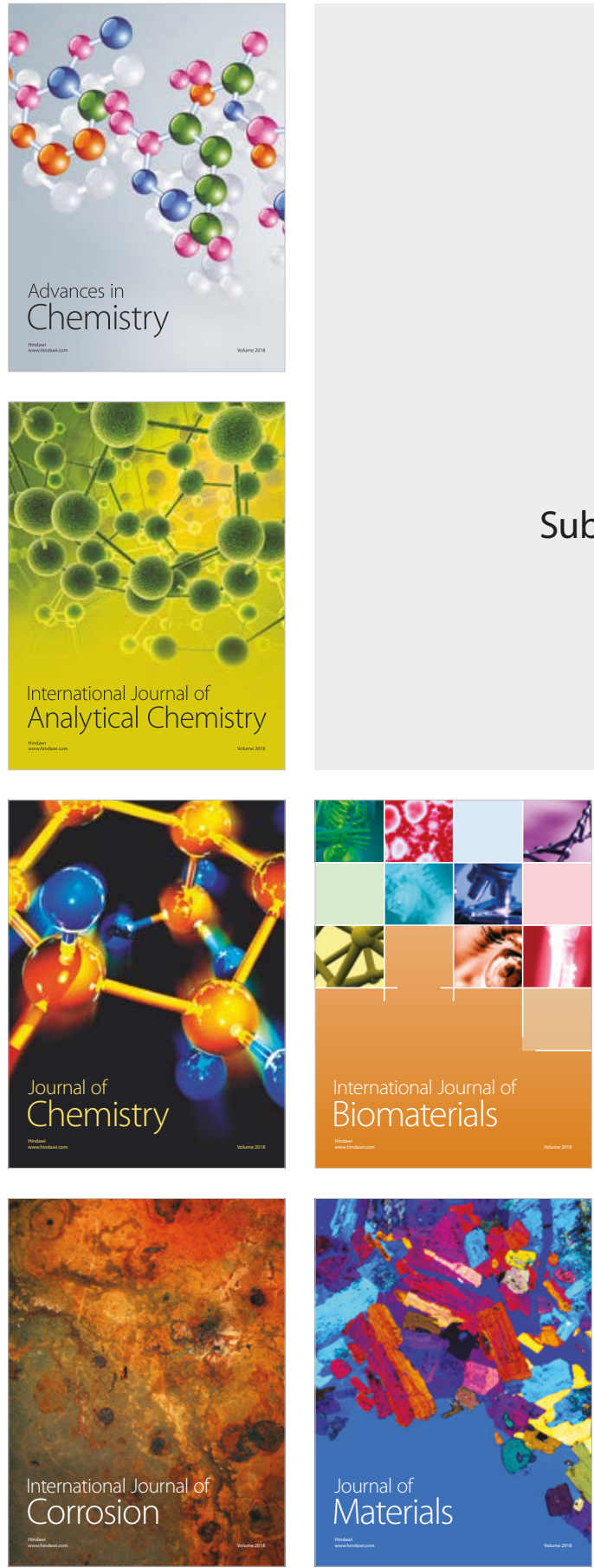

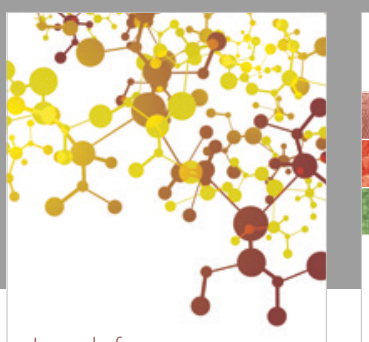

Journal of

Applied Chemistry
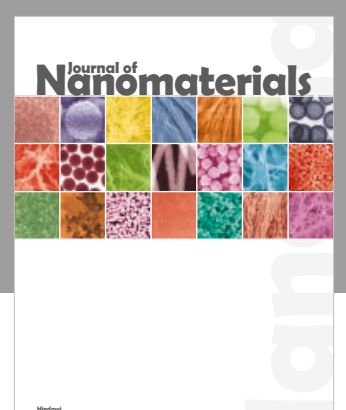

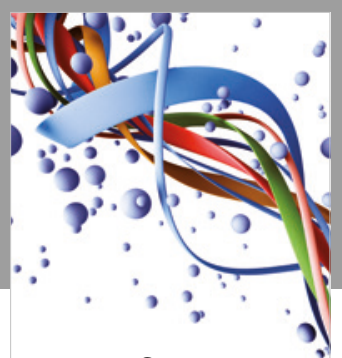

Scientifica

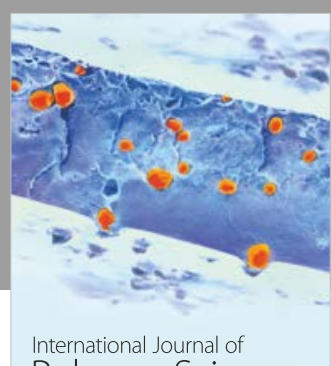

Polymer Science

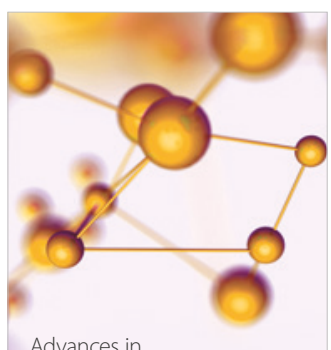

Physical Chemistry
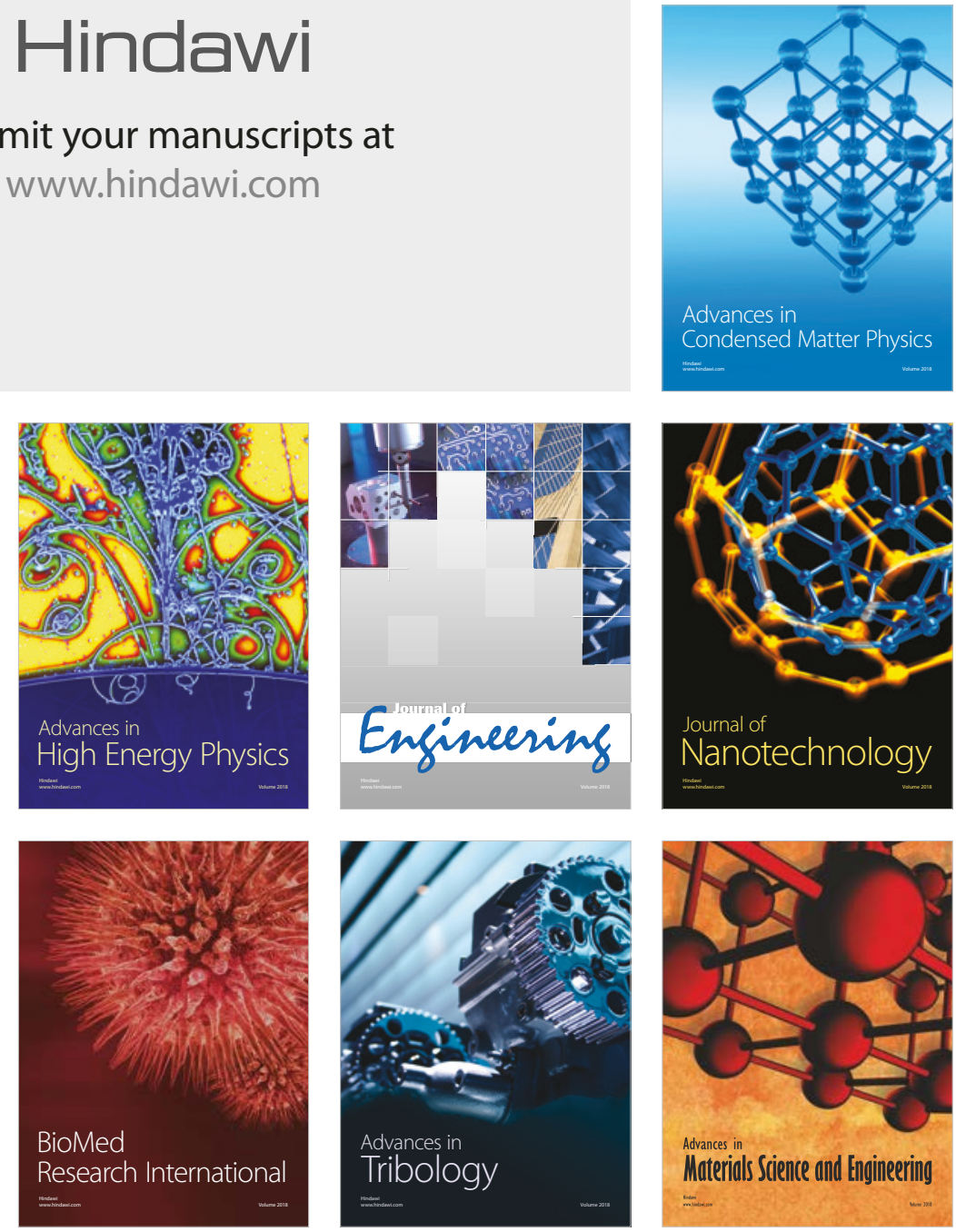